\title{
Aware of Life-Time in Lighting Applications by Applying PWM
}

\author{
Joy Iong-Zong Chen \\ Department of electrical Engineering Dayeh University \\ Changhua, Taiwan \\ E-mail:jchen@mail.dyu.edu.tw
}

\begin{abstract}
A contactless way to prolong the life-time of lighting applications by PWM (pulse width modulation) device power system is developed in this article. The system can match most sustainable energy, such as solar energy or wind power generator. It can automatically switch with the best way to control sustainable energy power system which fit in. On the other hand, because single chip can transfer power instantly to drive device up, like charge smartphone, it is a highly customize device. With the control system, traditional sustainable energy device can work much longer life-time and stable. Through adjustable wireless protocol, PWM, low-power multi-purpose regulator circuit and instant feedback display, so it can be optimized most sustainable energy devices. The proposed control system is built by a single chip microcontroller which is serving as the integration of the control core of the circuit, voltage feedback monitor at the same time. In order to improve system performance, the research made for different drive designs to analyze and compare, and simulated calculation and experimented.
\end{abstract}

Keywords: contactless, PWM, power system, single chip microcontroller.

\section{INTRODUCTION}

In recent, due to the population in the world is incremental fast and the speed of developing in science technology is quick, the life resources in the whole world which we rely on are much less gradually. How to look for new replacement of the energy resources becomes a serious issue for addressing, the green energy is especially. For example, some of the green energies which can be explored including bio-diesel, solar power, wind power etc.. Therefore, it is also a very important issue for discussion to apply the all new developed green energy efficiently. There are many research reports claimed such serious events already. In [1] explores the conditions under which the continuous price rise of renewable energy is restrained in the presence of technological progress in harnessing renewable energy. The results show that to sustain the age of cheap energy, the growth rate of technology in harnessing renewable energy has to be larger than the discount rate. In [2] presents preliminary work towards the development of a complete smart street lighting system focusing on Malaysian environment. The system integrates Wireless Sensor Network (WSN) concept in the development, utilizing Waspmote as a sensor node, Meshlium gateway and computer as a server. The research topic of [3] is to integrate

\author{
Jia hao Fang \\ Department of electrical Engineering Dayeh University \\ Changhua, Taiwan \\ E-mail:R0103016@mail.dyu.edu.tw
}

not only the green mode function but some useful functions such as LEB, OVP, soft start. Besides, the pulse-frequencymodulation (PFM) technique is usually adopted at light-load condition, and slope compensation. Consequently, the power loss of the switching regulator can be reduced by the proposed current mode PWM control IC. In [4], a dimmable light emitting diode (LED) driver with one cycle control (OCC) is proposed for low power lighting applications. LEDs are more energy efficient than the incandescent bulbs, halogen bulbs and even compact fluorescent light bulbs. In [5] the author Galigekere applied the method of PWM (pulse width modulation) to save the energy for an oscillation circuit contains with some capacitors and inductors that is working in CCM (continuous conduction mode). We inspect the efficiency caused by the whole application of small signal with transistor circuit proposed by Lian [6]. A DC-toDC convertor works out in [7] presents by Kim, which is to combine with a single pole three-phases circuit solves the problems and to solve the saving energy consuming in solar cell system problems [8-11]. The motivation of this article is arming in proposing and developing an energy saving system which applies the PWM schemes in deploying with a contactless wireless control method. Finally, the implemented system is verified by some experiences in laboratory and the simulation results are illustrating the energy saving fact.

\section{THE ARCHITECTURE OF CONTACTLESS PROGRAMMABLE PWM CONTROL SYSTEMS}

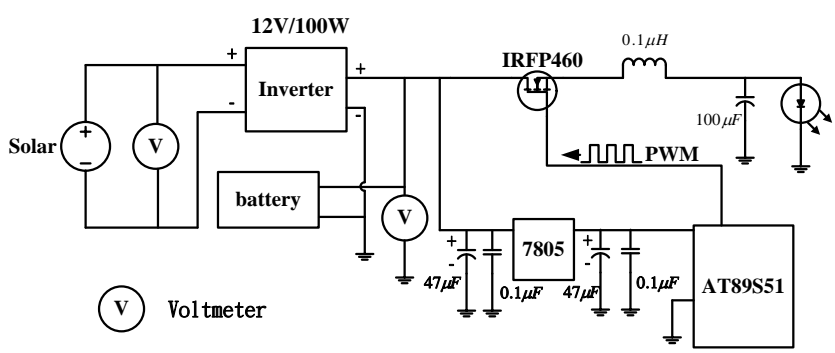

Figure 1. The schematic diagram of contactless programmable PWM control systems

The proposed contactless programmable PWM control system is shown in Fig. 1 in which includes solar cell plates, blue-tooth components, main controller with 8051 microchip, 
extended storage devices, chargeable batteries, and a DC-toDC converter.

In fact, strictly to announce there are two main subsystems for describing. Firstly, one of the sub-system is the part of hardware DC-to-DC convertor involves the voltage step-down circuit, and which is a MOSFET component used to joint together becomes the kernel of the step-down circuit. Besides, it tunes the work duty cycles which varies with the operation of PWM scheme [6]. The second one of the subsystem is a single chip with software controlling, and it is selecting 8051 single chip for dominating overall the control system. On the other hand, the later sub-system is charging in gathering and analyzing all of the data collected by the surrounding deployed sensing receiver nodes. On the basis of run out information the sub-system can give commands for controlling the next stage of the work of the system. The characteristics of popularity and the customization generated by the system causes the implemented energy saving system can suitable for every kind of green energy systems. Furthermore, it can be widely deployed in the utilization of power energy for following up different mankind behaviors [7]. For instance, the application of a solar cell which is embedded in a night lamp and the charging energy will be stored in battery when it is sunny weather. The PWM system will do give a command to the night lamp control device depends on the case of the weather. The command is with about just $30 \%$ of the energy illumination at evening PM5:00, and 100\% of the energy illumination at evening PM7:00. On contrary, the main controller will detect and report the efficiency of an energy storage device at raining day, and turn on the illustration function for night lamp control device.

\section{A. Single Chip Controller}

A single chip controller is combined with CPU (central processing unit), memory unit, and I/O (input/output) unit, all which reminded previously are product in an independent micro-computer system. Generally, it has small size, user friendly, ease wire, and scalability.

\section{B. Pulse Width Modulation (PWM)}

A technique with transferring the analog signal to pulse is described in this sub-section, and which is called as PWM. Accordingly, the turn out pulse signal are all with fixed duty cycle which normally changes to the amplitude of the analog signal. A widely applied technique which is not a build in for 8051 single chip, however, the PWM functions are generally by means of the counter/timer generation embedded in the single chip.

\section{Analog-to-Digital Converter}

It is known that some physic phenomenon, such as light, temperature, pressure, and movement etc. in the natural world, can be got for application the analog-to-digital transformation by sensors is necessary. The format of the turning out results may be voltage or current. Once the digital signals are obtained then passed into the processing with a micro-computer system for the purpose of surveillance, measuring, and recording [10]. There is an analog-to-digital device is adopted for recording and monitoring designed in this system.

$D$.

The voltage step-down controller is applied in the development of this control system, and the controller can adjust the output voltage to the load for saving the energy of battery. We deploy the specifications of a N-channel enhanced MOSFET for transmitting the output comes from PWM to the gate pole $(\mathrm{G})$ of the MOSFET, then which causes the voltage, Vds, between drain pole (D) and the source $(\mathrm{S})$ pole can be adjusted by the voltage, Vgs, between gate pole $(\mathrm{G})$ and the source $(\mathrm{S})$ pole. The positive property between Vds and Vgs can used to easily control the energy comes out from the battery [12].

\section{EXPERIMENTAL RESULTS}

Fig. 4 in which shows the photo of the fully implemented PWM control system aforementioned. We set up a deployment environment where are with a battery has the specification of 12 volts /8 amps-hour, the loading and the solar cellar are with 12 volts / 2.5 amps and 12 volts / 40 watt, respectively. All those implemented components are operating in a sunny weather day. The data includes voltage and current are collected per 10 minute in a day, while the battery is full charging and $100 \%$ energy output to the loading. For the purpose of validating the functionalities of the implemented system, the curves of life-time versus current values are shown in Fig. 2 .There are two cases with different loading deployed in the validation that are corresponding to 100 percentage loading and the other is with 70 percentage loading. It is reasonable to know that the life-time for the case of 100 percentage is much short than the other case. On the other hand, the decay rate of the supplied current for the 100 percentage loading is much fast than the one with 70 percentage loading. The power consuming in the devices embedded into the system is also one key point of parameter for prolonging the life-time of the implemented system. The results of the situation if the power supply for LCD display devices impact the life-time of the system is shown in Fig. 3.

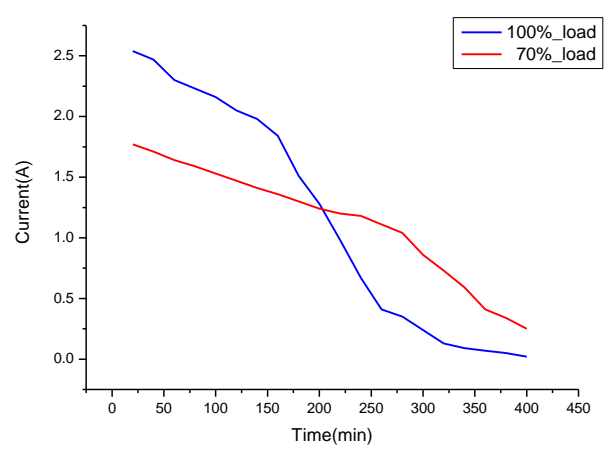

Figure 2. Life-time curves of cases with $100 \%$ vs $70 \%$ 


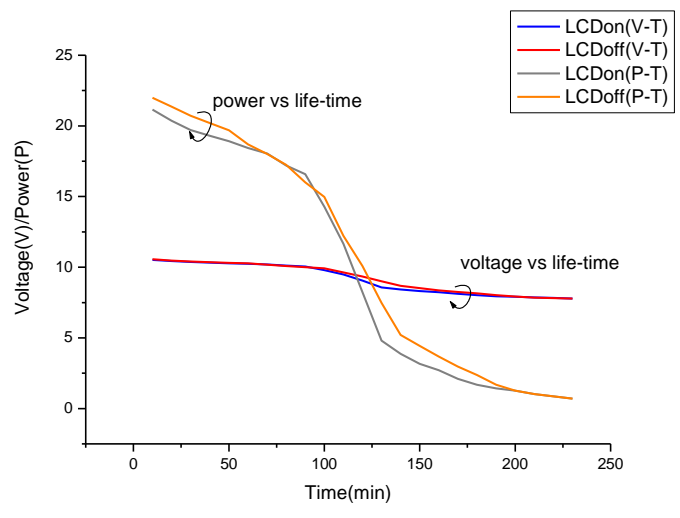

Figure 3. Life-time vs voltage and power for curves of cases with LCD on/off display

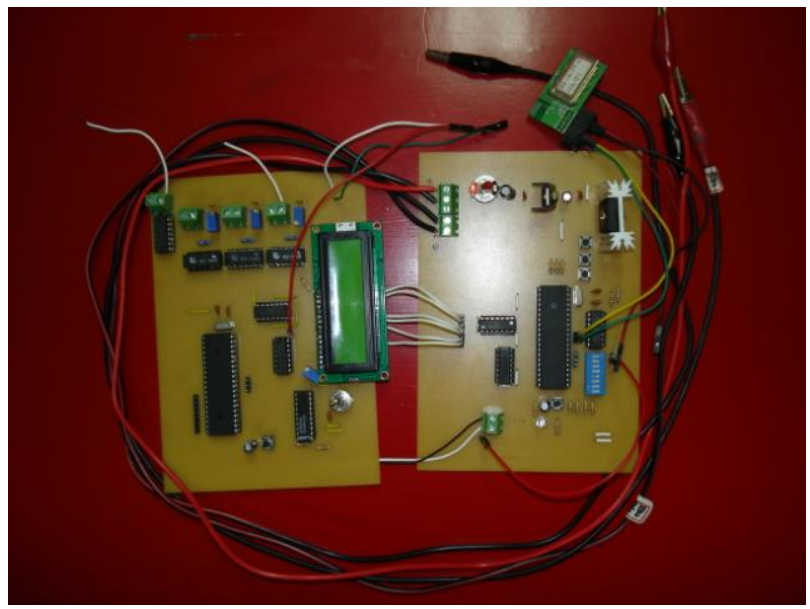

Figure 4. Solar billboard and the circuit of contactless programmable PWM control systems

There are two groups of curve for understanding the system performance with voltages and power dissipate versus life-time. It is clear to see that the life-time is sustainable about 250 minutes whatever the LCD display is under on or off case with the supplied voltage is always keep at 10 volts. The fact proves that the aware of life-time system implemented in this article is satisfied.

\section{CONCLUSION}

An energy saving control system applies PWM scheme is completely implemented in this article. The present technique for the proposed system is verified by 3 cases with experimental results. The contactless programmable PWM control system can not only enlarge the life-time of a battery embedded in a green energy system, but the efficiency of the green energy system can be monitored and analyzed. Furthermore, the output energy given to a loading can be adjusted by different kind of situations surrounding the environment. For the reason that the developed system is just a prototype, however, the experimental results have shown that the system can definitely save the energy. In the future, different kind of the applied transistors can be replaced with for the reason of low cost and low power consumption. By the way, an automatic scheduling algorithm can be adopted as the main controller, and which can adjust the adequate amount of energy output to loading according to power surplus.

\section{REFERENCES}

[1] Hazuki Ishida, Can Renewable Energy Sustain an Age of Cheap Energy?, Journal of Technology Innovations in Renewable Energy, 2013, pp.201-204.DOI: http://dx.doi.org/10.6000/1929-6002.2013.02.03.1.

[2] Yusoff, Y.M., Rosli, R., Karnaluddin, M.U., Samad, M.,Towards smart street lighting system in Malaysia Wireless Technology and Applications (ISWTA), IEEE Transactions on 2013,pp. 301-305.

[3] Hung-Da Hsu, Tsorng-Juu Liang, Bin-Da Liu, Kai-Hui Chen, Design of a green mode PWM controlIC,Circuits and Systems, 2008. APCCAS 2008. IEEE Asia Pacific Conference on 2008, pp.1876 1879

[4] Hariprasath, S., Balamurugan, R., A valley-fill SEPIC-derived power factor correction topology for LED lighting applications using one cycle control technique, Computer Communication and Informatics (ICCCI), International Conference on 2013, pp. 1- 4.

[5] Galigekere, Veda Prakash, Marian K. Kazimierczuk, Analysis of PWM z-source dc-dc converter in $\mathrm{ccm}$ for steady state, Circuits and Systems I: Regular Papers, IEEE Transactions on 59.4 ,2012, pp.854863.

[6] Lian, K. L., Derivation of a small-signal Harmonic Model for closedloop power converters based on the state-variable sensitivity method, Circuits and Systems I: Regular Papers, IEEE Transactions on $59.4,2012$, pp.833-845.

[7] Hongrae Kim, Parkhideh, B., Bongers, T.D., Reconfigurable solar converter: a single-stage power conversion PV-battery system, Heng Gao Power Electronics, IEEE Transactions on 28.8 ,2013, pp.37883797.

[8] Williams, Richard K., Multiple gated MOSFET for use in DC-DC converter, U.S. Patent 5,973,367. ,1999.

[9] Rashid, R.A., Yusoff, R., Bluetooth performance analysis in personal area network (PAN), RF and Microwave Conference. ,2006, pp.393397.

[10] Ho, C.Y.-F., Ling, B.W.-K., Yan-Qun Liu, Tam, P.K.-S., Kok-Lay Teo, Optimal PWM control of switched-capacitor DC-DC power converters via model transformation and enhancing control techniques, Circuits and Systems I: Regular Papers, IEEE Transactions on $55.5,2008$, pp.1382-1391.

[11] Ismail, E.H., Al-Saffar, M.A., Sabzali, A.J., Fardoun, A.A., A family of single-switch PWM converters with high step-up conversion ratio, Circuits and Systems I: Regular Papers, IEEE Transactions on $55.4,2008$, pp.1159-1171.

[12] Ming -feng Yang, GOTOP Information Inc, ISBN 978-986-276-2639. 